\title{
ON MULTIPLE SALIÉ SUMS
}

\author{
W. DUKE
}

(Communicated by William Adams)

\begin{abstract}
This note shows that the Davenport-Hasse relation for Gauss sums is equivalent to the evaluation of some multidimensional exponential sums that generalize that of Salié.
\end{abstract}

This paper shows that the Davenport-Hasse relation for Gauss sums is equivalent to the evaluation of some multidimensional exponential sums that generalize that of Salié. In addition to being interesting in their own right, these sums also occur in the analysis of Fourier expansions of certain Poincaré series on the $n$-fold cover of $G L(n)$. For example, if one considers the analogues of the exponential sums in Theorem 1 of M. Larsen's appendix to [B-F-G] that occur for the Poincare series on the 3-fold cover of $G L(3)$, the hyper-Kloosterman sums that arise are of the type considered here when $n=3$. Thus the evaluation given below may turn out to be important in the further development of the theory of such automorphic forms when $n \geq 3$. This is certainly the case for $n=2$, as the work of Iwaniec [I] shows.

Let $F=G F(q), q=p^{r}$ for $p$ prime, and $n \in \mathbf{Z}^{+}$be such that $q \equiv 1$ $(\bmod n)$. For $\psi$ a multiplicative character of order $n$ and $a \in F^{*}$ consider the $n$-dimensional Salié sum defined by

$$
S_{n}(a)=\sum_{\substack{x_{1} x_{2} \cdots x_{n}=a \\ x_{i} \in F^{*}}} \psi\left(x_{2} x_{3}^{2} \cdots x_{n}^{n-1}\right) e_{q}\left(x_{1}+\cdots+x_{n}\right),
$$

where $e_{q}(x)=e(\operatorname{tr} x / p)$. (Note that $S_{n}(a)$ does not depend on the choice of $\psi$.)

\section{Theorem.}

$$
S_{n}(a)=\varepsilon_{q, n} q^{(n-1) / 2} \sum_{x: x^{n}=a} e_{q}(n x)
$$

where

$$
\varepsilon_{q, n}= \begin{cases}1 & \text { if } n \text { is odd } \\ (-1)^{(q-1)(n-2) / 8+r-1} i^{(p-1)^{2} r / 4} & \text { if } n \text { is even. }\end{cases}
$$

Received by the editors September 13, 1990.

1980 Mathematics Subject Classification (1985 Revision). Primary 11T21; Secondary 11L05, $11 \mathrm{F55}$.

Research supported by NSF Grant DMS-8902992 and a Henry Rutgers Research Fellowship. 
In fact $(*)$ is equivalent to the Davenport-Hasse relation, which states that for any multiplicative character $\chi$

$$
\tau(\chi) \tau(\chi \psi) \cdots \tau\left(\chi \psi^{n-1}\right)=\tau\left(\chi^{n}\right) \bar{\chi}^{n}(n) \tau(\psi) \tau\left(\psi^{2}\right) \cdots \tau\left(\psi^{n-1}\right)
$$

where $\tau(\chi)=\sum_{x \in F^{*}} \chi(x) e_{q}(x)$. For $\chi$ with $\chi^{n}=1$ this formula is trivial, and otherwise it was obtained by Davenport and Hasse in [D-H] (see also [L, p. 61]). In case $n=3$ and $q=p$ a proof of $(* *)$ that parallels one for the triplication formula for the $\Gamma$-function has been given by Greene and Stanton [G-S].

To prove $(*)$ first multiply both sides of $(* *)$ by $\bar{\chi}(a)$ and sum over $\chi$, i.e., take the Fourier transform. This yields

$$
S_{n}(a)=\tau(\psi) \tau\left(\psi^{2}\right) \cdots \tau\left(\psi^{n-1}\right) \sum_{x: x^{n}=a} e_{q}(n x) .
$$

Now $(*)$ follows from the classical formulas

$$
\begin{array}{ll}
\tau\left(\psi^{k}\right) \tau\left(\psi^{n-k}\right)=\psi^{k}(-1) q, & k=1, \ldots, n-1, \\
\tau(\varphi)=(-1)^{r-1} i^{(p-1)^{2} r / 4} q^{1 / 2}, & \text { for } p>2,
\end{array}
$$

where $\varphi$ is of order 2 , together with the fact that $\psi(-1)=-1$ iff $n$ is even and $(q-1) / n$ is odd (see for example [L-N, Theorem 5.15 and Remark 5.13]). The converse is similar.

In the case of $n=2$ this yields Salié's original result [S] (Salié worked with $q=p)$

$$
\sum_{x} \varphi(x) e_{q}(x+a \bar{x})=\varepsilon_{q, 2} \sqrt{q} \sum_{x: x^{2}=a} e_{q}(2 x),
$$

while for $n=3$ it gives

$$
\sum_{x_{1} x_{2} x_{3}=a} \psi\left(x_{2} x_{3}^{2}\right) e_{q}\left(x_{1}+x_{2}+x_{3}\right)=q \sum_{x: x^{3}=a} e_{q}(3 x) .
$$

We remark that many other multiple exponential sums can be similarly treated, for example those studied by Mordell in [M]. Also, $S_{3}\left(a^{3}\right)$ was independently evaluated by $\mathrm{N}$. Elkies (unpublished) by an elegant argument using elliptic curves.

\section{ACKNOWLEDGMENTS}

I would like to thank Dan Bump for pointing out the automorphic occurrence of $S_{n}(a)$. Thanks also to Ilan Vardi for several helpful suggestions.

\section{REFERENCES}

[B-F-G] D. Bump, S. Friedberg, and D. Goldfeld, Poincaré series and Kloosterman sums for $S L(3, \mathrm{Z})$, Acta Arith. 50 (1988), 31-89.

[B-H] D. Bump and J. Hoffstein, Cubic metaplectic forms on $G L(3)$, Invent. Math. 84 (1986), 481-505.

[D-H] H. Davenport and H. Hasse, Die Nullstellen der Kongruenzzetafunktionen in gewissen zyklischen Fällen, J. Reine Angew. Math. 172 (1934), 151-182.

[G-S] J. Greene and D. Stanton, The triplication formula for Gauss sums, Aequationes Math. 30 (1986), 134-141.

[I] H. Iwaniec, Fourier coefficients of modular forms of half-integral weight, Invent. Math. 87 (1989), 385-401. 
[L] S. Lang, Cyclotomic fields, Springer-Verlag, New York, 1978.

[L-N] R. Lidl and H. Niederreiter, Finite fields, Addison-Wesley, Reading, MA, 1983.

[M] L. J. Mordell, On some exponential sums related to Kloosterman sums, Acta Arith. 21 (1972), 65-69.

[S] H. Salié, Über die Kloostermanschen Summen $S(u, v ; q)$, Math. Z. 34 (1932), 91-109.

[W] K. S. Williams, Note on Salié's sum, Proc. Amer. Math. Soc. 30 (1971), 393-394.

Department of Mathematics, Rutgers University, New Brunswick, New Jersey 08903 